\title{
Chemotherapy Negates the Effect of SDF1 mRNA to Distant Metastasis and Poor Overall Survival in Breast Cancer Patients
}

\author{
Kristanto Yuli Yarso ${ }^{1}$, Monica Bellynda ${ }^{2 *}$, Akhmad Azmiardi ${ }^{3}$, Brian Wasita ${ }^{4}$, \\ Didik Setyo Heriyanto ${ }^{5}$, Indwiani Astuti ${ }^{6}$, Mohammad Hakimi ${ }^{7}$, Teguh Aryandono ${ }^{8}$
}

\begin{abstract}
Objective: Investigate the effect of SDF1a, nuclear, and cytoplasmic CXCR4 breast cancer tissue on metastasis and overall survival in patients with complete-chemotherapy and no-chemotherapy. Methods: Cohort ambidirectional design was employed with survival analysis that followed the patient's diagnosis until obtaining the outcome, distant metastasis, or death. We analyzed samples in three groups (all-patient, no-chemotherapy, and complete-chemotherapy groups). Breast cancer cell nuclear and cytoplasm expressions of CXCR4 protein were examined using immunohistochemistry. Amplification of mRNA SDF1a of breast cancer tissue was examined using rtPCR on 131 samples from the same initial paraffin block. Results: In the distant metastasis and Overall Survival (OS) analysis, there was no correlation between cytoplasmic and nuclear CXCR4 in all-patient, no-chemotherapy, and complete-chemotherapy groups. SDF1a was significantly correlated to shorter distant metastasis and poor OS in the all-patient ( $p=0.004$ and $p=0.04$, respectively) and no-chemotherapy group ( $\mathrm{p}=0.008$ and $\mathrm{p}=0.026$, respectively). However, in the complete-chemotherapy group, SDF1a was not correlated to either metastasis $(\mathrm{p}=0.527)$ or OS $(\mathrm{p}=0.993)$, advanced stage demonstrated a strong association on shorter distant metastatic in no-chemotherapy $(p=0.021)$ and complete-chemotherapy group $(p=0.004)$ and also poor OS in both groups ( $p=0.006$ and $p=0.002$, respectively). The hormone receptor showed a protective effect on the no-chemotherapy group's OS $(p=0.019)$. Meanwhile, not undergoing chemotherapy was associated with poor OS in the all-patient group ( $\mathrm{p}=0.011)$. Conclusion: SDF1a mRNA amplification has a significant correlation with the occurrence of metastasis and OS in all-patient and no-chemotherapy group. Undergoing chemotherapy negates the effect of SDF1a for distant metastasis and OS.
\end{abstract}

Keywords: Nuclear CXCR4- cytoplasmic CXCR4- mRNA SDF1a- breast cancer- metastasis- chemotherapy

Asian Pac J Cancer Prev, 22 (3), 757-766

\section{Introduction}

Breast cancer is a health problem in women around the world. In 2018, it was the second common disease with the fifth-highest mortality worldwide. Total cases of breast cancer were 2,088,849 in 2018. Indonesia ranked first with a total of 58,256 patients (WHO, 2019). In Denpasar, Indonesia, more than 90 new cases of breast cancer are found each year. From the data of 678 patients, more than $43 \%$ of breast cancer patients are in local advanced stage III, while 26\% have metastases (Yarso et al., 2012).

One crucial component of metastasis is the axis stromal cell-derived factor $1 \alpha$ (SDF1a) with its receptor CXCR4 (Chemokine Motif Receptor 4). Some studies suggest that SDF1a from the cancer microenvironment will spur cancer cells to migrate and associated with metastasis (Kang et al., 2009; Liu et al., 2009). Others stated that SDF1a holds cancer cells in tumor tissue and prevents metastasis (Mirisola et al., 2009).

In a Korean study, high expression of CXCR4 on membranes, cytoplasm, and breast cancer cell nuclei was associated with younger age, large tumor size, and poor OS. Another study has shown that nuclear CXCR4 expression and lymph node metastases are associated with the prognosis of breast cancer patients. Several studies have shown that $C X C R 4$ expression in the membranes is associated with prognostic factors, but nuclear $C X C R 4$ expression is not (Woo et al., 2008; Kim et al., 2009; 
Yasuoka et al., 2008). CXCR4 cycle after exposure to high SDF1a results in endocytosis and is in the cytoplasm through an internalization process. This CXCR4 will then be ubiquitinated (Bushillo et al., 2010). Some of these clinical studies show the effects of SDF1a and CXCR4 are not the same and are inconstant. In this study, we investigate chemotherapy as a factor influencing the effect of SDF1a and CXCR4 on distant metastasis and OS.

\section{Materials and Methods}

\section{Patients and tissue samples}

The data were obtained from dr. Moewardi and Kasih Ibu Hospital in Surakarta, Central Java, Indonesia. The patients' data were collected from January 2013 to January 2015. The inclusion criteria are (a) patients with stage 1-3 breast cancer at the time of diagnosis who visited the oncology clinic, (b) patients who could be tracked and followed up via direct or phone interview, and c) patients with accessible paraffin blocks. We observed 131 subjects. Metastasis events were recorded from the diagnosis until they showed symptoms and had distant metastases or deceased. Observations were conducted prospectively until January 2020 for 1-135 months with a median follow-up of 27 months. The examination or tracing of metastasis was performed on schedule or if there was any symptom of metastasis. The subjects who did not show up for examination were examined at home. Observation and recording of the signs of metastasis and death were undergone both in person and through phone interviews. All data were taken from medical records on the baseline and follow-up time.

According to ASCO, immunohistochemistry (IHC) examination with negative ER PR is if the number of stained nuclei in tumor cells is less than $1 \%$, and Her2 negative is if the number of stained nuclei is two or less with negative FISH. The appropriate cut-off point for Ki67 was determined negative if the value is less than $20 \%$ (Arima et al., 2019). There were 68 subjects (51.9\%) in the complete-chemotherapy group and 63 subjects in the no-chemotherapy group (49.1\%). The no-chemotherapy group consists of patients who refused chemotherapy, including patients dropping out of chemotherapy before four sessions for any reason. The chemotherapy regimen used was anthracycline base.

All patients underwent modified mastectomy surgery if possible. Radiation data cannot be analyzed because many patients did not receive the treatment or the therapy was out of the specified schedule for some reason.

The degree of differentiation is determined based on the Scarff-Bloom-Richardson system and classified into three grades (Fitzgibbons et al., 2000). Follow-up was assessed from the time of diagnosis to distant metastases and decease. OS, distant metastases, and locoregional recurrence were calculated in months, determined with clinical, pathological, and radiological data.

\section{Rt PCR and IHC}

Hematoxylin-eosin slides from the diagnosis were reviewed. The part containing the tumor was resected by $4 \mu$ and examined according to protocol with the primary
SDF-1 prepared before. The rt-PCR examination for SDF1 was performed on paraffin blocks from biopsy results. The paraffin blocks were stored at room temperature with age $<5$ years. To ensure the quality of the mRNA, we did concentration checking before further execution. We used spin column-based nucleic acid purification as the mRNA extraction method. The rt-PCR SDF1a examination results were classified into two groups, high and low, based on median values.

The mRNA samples were tested using the NEXproTM qRT-PCR Master Mix (SYBR) kit following the manufacturer's instructions. Quantitative PCR was carried out using Bioneer ExicyclerTM 96 Real-Time Quantitative Thermal Block. The PCR condition was as recommended by the manufacturer. The PCR primers were as follows: forward 5'-CAGAGCCAACGTCAAGCA-3' and reverse 5'-AGGTACTCTTGGATCCAC-3.

For CXCR4 examination, an IHC paraffin block was cut by $4 \mu$ and stained according to the CXCR 4 protocol. We used antibodies from $\mathrm{R}$ and $\mathrm{D}$ Human CXCR4 Antibody Monoclonal Mouse IgG2B Clone \# 44716 Catalog Number: MAB172. The test was performed from the same paraffin block as the SDF1 mRNA sample. The CXCR4 test was analyzed using two methods: the expression on the nucleus the cytoplasm. The assessment performed using scores calculated with the Image $\mathbf{J}$ program was divided into high and low expressions groups (Figure 1).

Digital images were captured using an Olympus CX21 microscope (Olympus Corporation, Tokyo, Japan) equipped with $10 \times, 20 \times$, and $40 \times$ objective lenses and an Optilab digital color camera (Miconos, Yogyakarta, Indonesia). The images were stored using an uncompressed image file format (bitmap). For every imaging session, an image from an empty slide background area was acquired (blank field image) to correct image color balance and uneven illumination. CXCR4 expression was analyzed using Image J Software with IHC profiler plugin according to Varghese et al. (2014) and score as negative (0), low positive $(1+)$, positive $(2+)$, and high positive $(3+)$. Score $0-2$ were categorized as low expression, and score 3 was categorized as high expression.

\section{Statistical Analysis}

Patients were followed up and documented from the time of diagnosis until distant metastasis or death. The survival and distant metastasis time of the high and low expression groups was determined using Kaplan-Meier survival analysis, and the characteristic data of both groups were analyzed using the chi-square Fischer exact test when applicable. The survival chart is presented in log-rank. Cox proportional hazards model and logistic regression analysis were used to evaluate the prognostic significance of each variable in the univariate and multivariate analyses. The analysis was conducted on all-patient group followed by no-chemotherapy and chemotherapy groups.

All statistical analyses were performed using SPSS Statistics software version 22.0 (IBM Corp., Armonk, $\mathrm{NY}$, USA). It is statistically significant if the result is less than 0.05 . 


\section{Results}

From the histological examination, the pathological diagnosis was invasive ductal carcinoma (IDC) in 116 patients $(88.4 \%)$, invasive lobular carcinoma (ILC) in 3 patients $(2.3 \%)$, medullary in 5 patients $(3.9 \%)$, mucinous in 1 patient $(0.8 \%)$, papillary in 3 patients $(2.3 \%)$, and scirrhous in 3 patients $(2.3 \%)$. The subject's clinicopathological characteristic is described more in Table 1.

\section{Expression of CXCR4 and SDF1a}

There was no $C X C R 4$ expression on cell membranes. High expression of nuclear CXCR4 was noted in 91 subjects, but 37 others had low expression. There were 17 positive and 111 negative cytoplasmic $C X C R 4$ expressions. Three IHC samples could not be read. The value of SDF1a amplification ranged from 1 to 11942 with a median of 34. We found 67 samples with high and 64 with low SDF1a amplification.

There was a correlation between high nuclear CXCR4

Table 1. Clinicopathological Characteristics in Patients According to the Expression of SDF1a, Cytoplasmic CXCR4 and nuclear CXCR4

\begin{tabular}{|c|c|c|c|c|c|c|c|c|c|c|c|c|}
\hline \multirow[t]{2}{*}{ Variable } & \multicolumn{4}{|c|}{ SDF1a } & \multicolumn{4}{|c|}{ Cytoplasmic CXCR 4} & \multicolumn{4}{|c|}{ Nuclear CXCR4 } \\
\hline & $\mathrm{N}$ & Low & High & $\mathrm{P}$ & $\mathrm{N}$ & Low & High & $\mathrm{P}$ & $\mathrm{N}$ & Low & High & $\mathrm{P}$ \\
\hline \multicolumn{13}{|l|}{ Age } \\
\hline$<50$ & $64(49 \%)$ & $30(47 \%)$ & $34(53 \%)$ & 0.73 & $63(49 \%)$ & $57(90 \%)$ & $6(10 \%)$ & 0.30 & $63(49 \%)$ & $17(27 \%)$ & $46(73 \%)$ & 0.70 \\
\hline$>50$ & $67(51 \%)$ & $34(51 \%)$ & $33(49 \%)$ & & $65(51 \%)$ & $54(83 \%)$ & $11(17 \%)$ & & $65(51 \%)$ & $20(31 \%)$ & $45(69 \%)$ & \\
\hline \multicolumn{13}{|l|}{ Stage } \\
\hline I, II & $44(34 \%)$ & $24(55 \%)$ & $20(45 \%)$ & 0.36 & $43(34 \%)$ & $39(91 \%)$ & $4(9 \%)$ & 0.42 & $43(34 \%)$ & $8(19 \%)$ & $35(81 \%)$ & 0.01 \\
\hline III & $87(66 \%)$ & $40(46 \%)$ & $47(54 \%)$ & & $85(66 \%)$ & $72(85 \%)$ & $13(15 \%)$ & & $85(66 \%)$ & $29(34 \%)$ & $56(66 \%)$ & \\
\hline \multicolumn{13}{|l|}{ Chemotherapy } \\
\hline Complete & $68(52 \%)$ & $37(54 \%)$ & $31(46 \%)$ & 0.22 & $66(52 \%)$ & $55(83 \%)$ & $11(17 \%)$ & 0.30 & $66(52 \%)$ & $21(32 \%)$ & $45(68 \%)$ & 0.56 \\
\hline Incomplete & $63(48 \%)$ & $27(43 \%)$ & $36(57 \%)$ & & $62(48 \%)$ & $56(90 \%)$ & $6(10 \%)$ & & $62(48 \%)$ & $16(26 \%)$ & $46(74 \%)$ & \\
\hline \multicolumn{13}{|c|}{ Hormone Status } \\
\hline Negative & $83(65 \%)$ & $37(45 \%)$ & $46(55 \%)$ & 0.19 & $81(64 \%)$ & $69(85 \%)$ & $12(15 \%)$ & 0.41 & $81(64 \%)$ & $24(30 \%)$ & $57(70 \%)$ & 0.84 \\
\hline Positive & $45(35 \%)$ & $26(58 \%)$ & $19(42 \%)$ & & $45(36 \%)$ & $41(91 \%)$ & $4(9 \%)$ & & $45(36 \%)$ & $12(27 \%)$ & $33(73 \%)$ & \\
\hline \multicolumn{13}{|l|}{ HER-2 } \\
\hline Negative & $70(55 \%)$ & $33(47 \%)$ & $37(53 \%)$ & 0.72 & $70(56 \%)$ & $63(90 \%)$ & $7(10 \%)$ & 0.42 & $70(56 \%)$ & $18(26 \%)$ & $52(74 \%)$ & 0.44 \\
\hline Positive & $58(45 \%)$ & $30(52 \%)$ & $28(48 \%)$ & & $56(44 \%)$ & $47(84 \%)$ & $9(16 \%)$ & & $56(44 \%)$ & $18(32 \%)$ & $38(68 \%)$ & \\
\hline \multicolumn{13}{|l|}{ KI67 } \\
\hline Negative & $48(37 \%)$ & $20(42 \%)$ & $28(58 \%)$ & 0.27 & $45(36 \%)$ & $43(96 \%)$ & $2(4 \%)$ & 0.05 & $45(36 \%)$ & $15(33 \%)$ & $30(67 \%)$ & 0.41 \\
\hline Positive & $81(63 \%)$ & $43(53 \%)$ & $38(47 \%)$ & & $81(64 \%)$ & $67(83 \%)$ & $14(17 \%)$ & & $81(64 \%)$ & $21(26 \%)$ & $60(74 \%)$ & \\
\hline \multicolumn{13}{|l|}{ Grade } \\
\hline I & $2(2 \%)$ & $2(100 \%)$ & $0(0 \%)$ & 0.35 & $2(2 \%)$ & $2(100 \%)$ & $0(0 \%)$ & 0.54 & $2(2 \%)$ & $0(0 \%)$ & $2(100 \%)$ & 0.28 \\
\hline II & $35(27 \%)$ & $18(51 \%)$ & $17(49 \%)$ & & $34(27 \%)$ & $31(91 \%)$ & $3(9 \%)$ & & $34(27 \%)$ & $13(38 \%)$ & $21(62 \%)$ & \\
\hline III & $91(71 \%)$ & $44(48 \%)$ & $47(52 \%)$ & & $91(72 \%)$ & 77 (85\%) & $14(15 \%)$ & & $91(72 \%)$ & $24(26 \%)$ & $67(74 \%)$ & \\
\hline
\end{tabular}

\section{Low High}

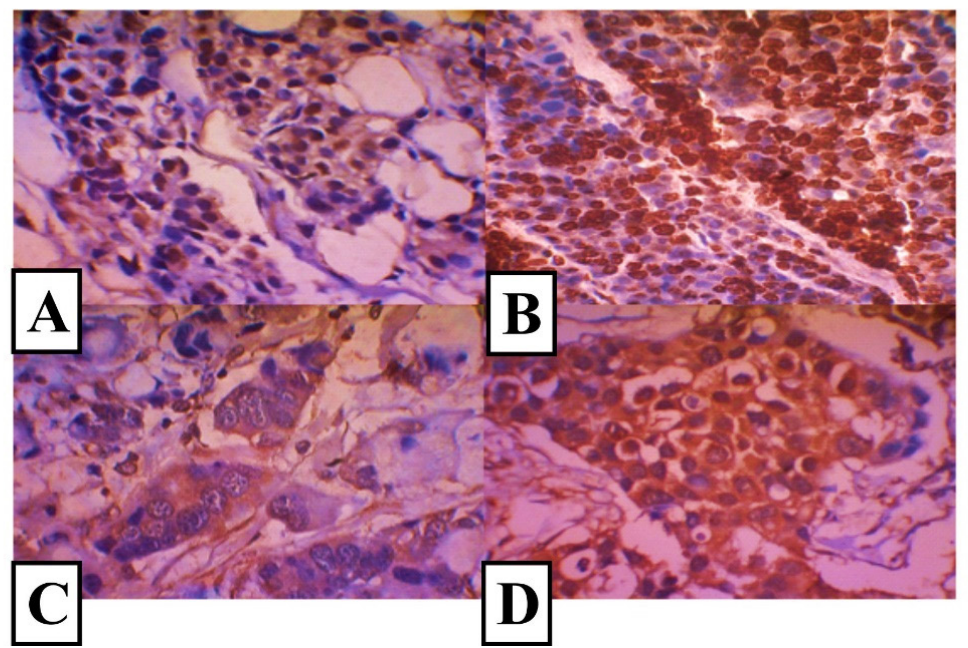

\section{Nuclear \\ expression}

\section{Cytoplasmic}

expression

Figure 1. (a). Low nuclear expression, (b). High nuclear expression, (c). Low cytoplasmic expression, (d) High cytoplasmic expression 

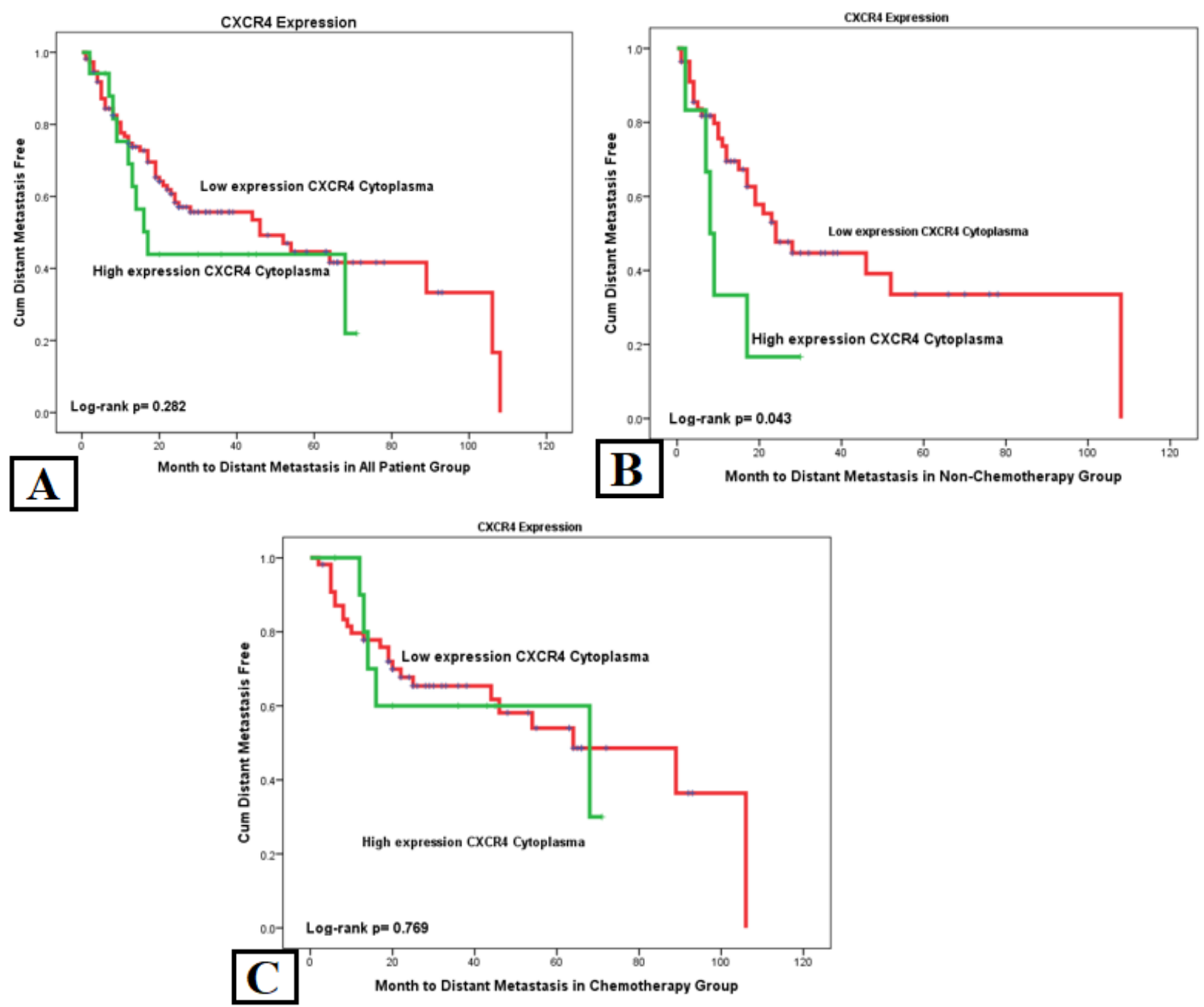

Figure 2. Time from Diagnosis to Distant Metastasis Determined by Cytoplasmic CXCR4. (a) In the all-patient group, the cytoplasmic CXCR4 expression showed a non-significant to correlation to metastasis. (b) However, the nonchemotherapy group showed a significant difference in high cytoplasmic CXCR4 expression with a worse metastasis. (c) Meanwhile, in the complete-chemotherapy group, there was no difference in high and low cytoplasmic CXCR4.

and advanced stage, between high cytoplasmic CXCR4 and positive KI67 in bivariate analysis. More than half $(51,5 \%)$ of the subjects had distant metastasis to the lung, bone, liver, contralateral breast, brain, and others by $25.2 \%, 8.4 \%, 5.3 \%, 4.6 \%, 2.3 \%$, and $3.1 \%$, respectively. Local recurrence had been noted on $7.6 \%$ of the subjects. There were 68 (51.9\%) subjects who completed chemotherapy, while $63(48.1 \%)$ of them dropped out of or did not undergo chemotherapy.

\section{Distant Metastasis}

We used the Kaplan-Meier survival curve for distant metastasis based on each marker in the all-patient group. There was a significant difference found in the SDF1a examination of metastasis with log-rank $\mathrm{p}=0.004 \mathrm{HR}=$ 1.903. Another significant variable was stage with $\mathrm{p}=0.000 \mathrm{HR}=3.966$ (Table 2). It means high SDF1 and advanced stage was associated with shorter time of distant metastasis. High cytoplasmic CXCR4 expression and SDF1a amplification was also associated with shorter time to distant metastasis in no-chemotherapy group (Figures 2 and 3).
To ensure the effect of chemotherapy on the results of this study, we conducted an analysis of samples divided into two groups, no-chemotherapy and complete-chemotherapy groups. Results showed that SDF1a has a strong correlation in the no-chemotherapy group with $\mathrm{p}=0.008 \mathrm{HR}=3.039$ but had no correlation in the complete-chemotherapy group. Another significant variable in the no-chemotherapy group is the stage with $\mathrm{p}=0.021 \mathrm{HR}=3.534$. There were no significant variables for the complete-chemotherapy group, except for stage with $\mathrm{p}=0.004 \mathrm{HR}=3.707$, which is a constant variable affecting distant metastasis (Table 2).

\section{Overall Survival}

Analysis of SDF1a with OS in the all-patient group showed a correlation with $\mathrm{p}=0.040$. Another significant variable was chemotherapy with $\mathrm{p}=0.011 \mathrm{HR}=2.168$ and stage with $\mathrm{p}=0.002 \mathrm{HR}=3.870$. We analyzed two groups of chemotherapy to eliminate the confounding effect. In the no-chemotherapy group, SDF1a showed a correlation with $\mathrm{p}=0.026 \mathrm{HR}=2.738$ and stage with $\mathrm{p}=0.006 \mathrm{HR}=6.203$. Another variable, the hormone 

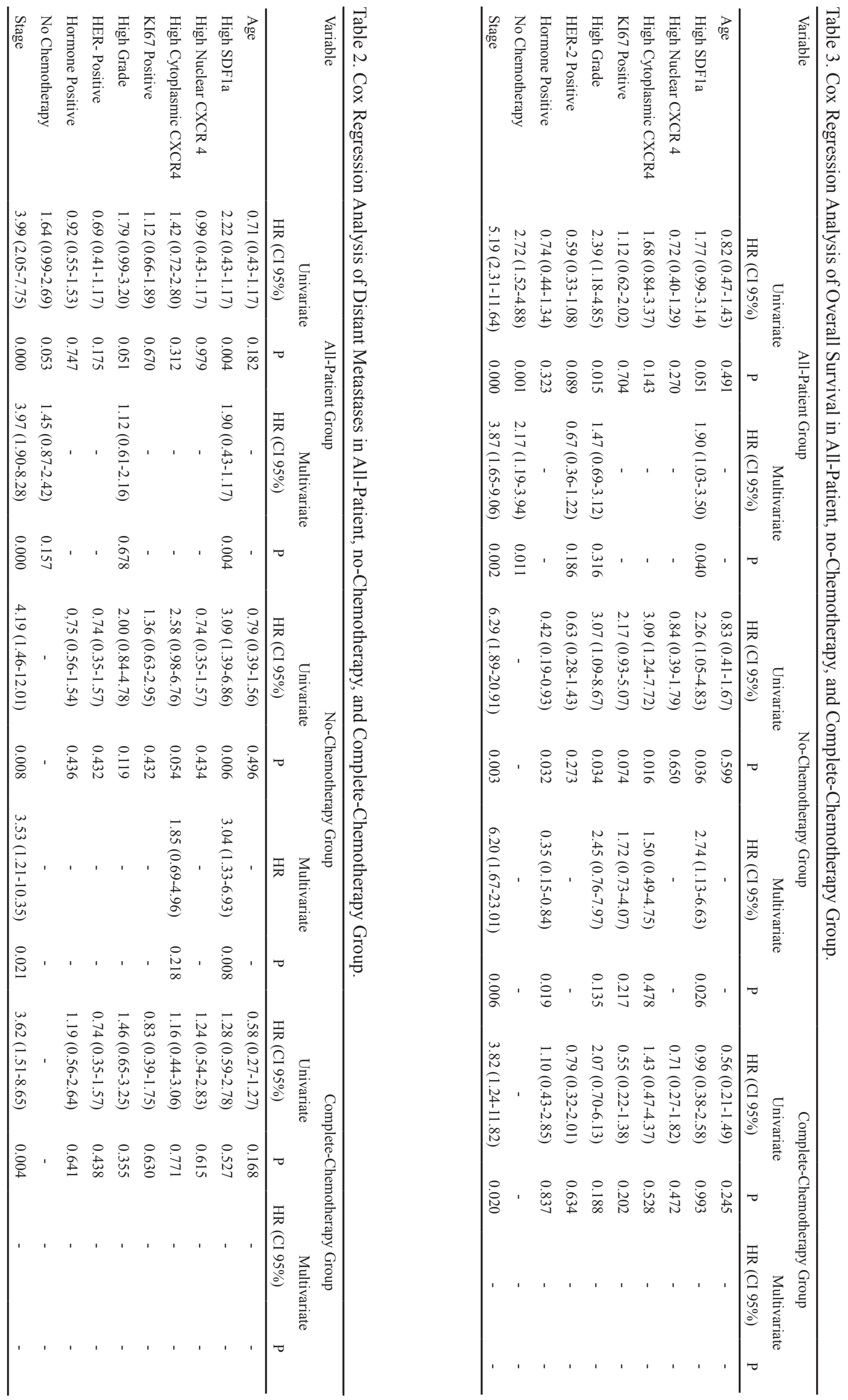

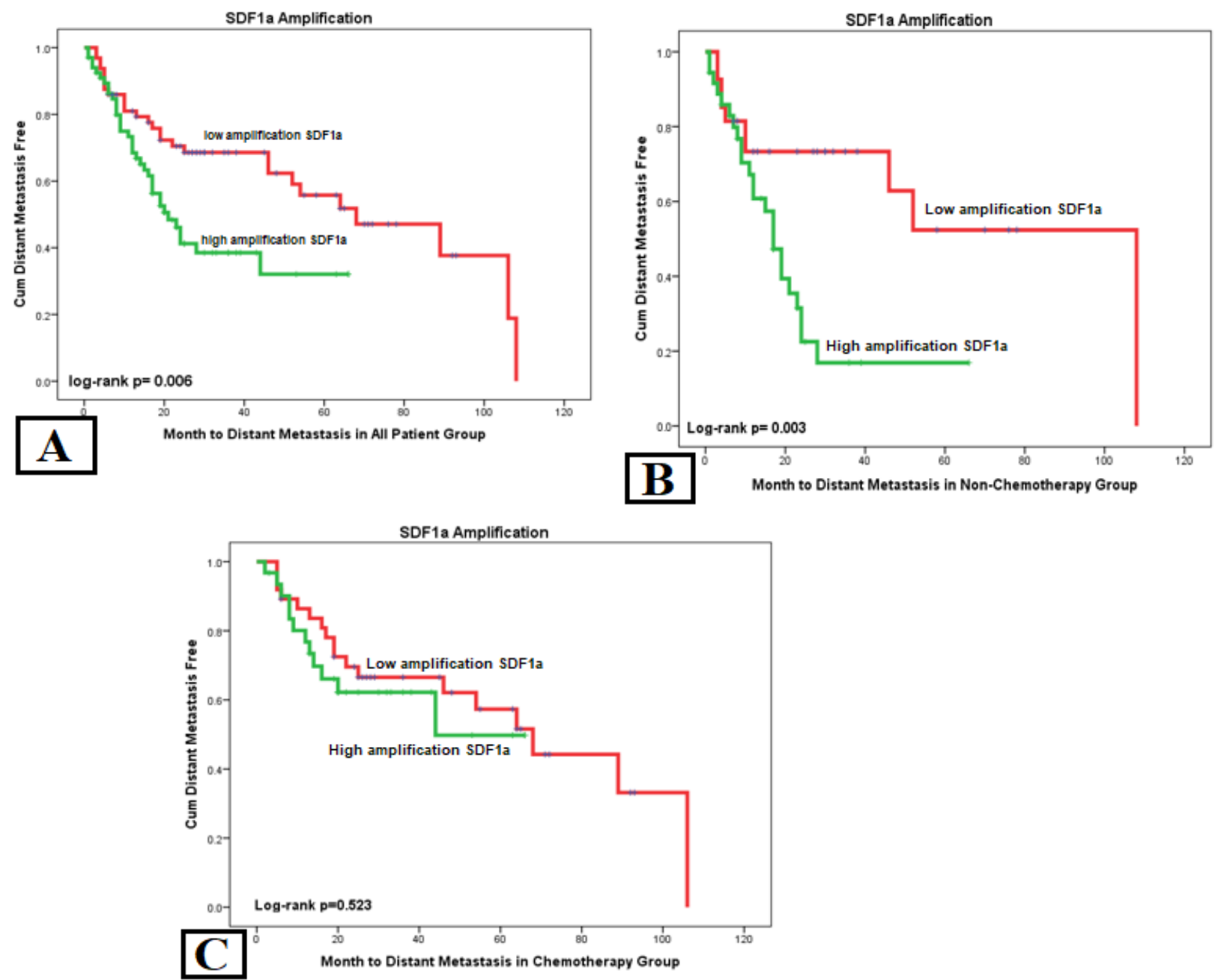

Figure 3. Time from Diagnosis to Distant Metastasis Determined by SDF1a. (a) In the all-patient group, SDF1a high amplification showed a significantly shorter metastasis time. (b) Likewise, the non-chemotherapy group showed a significant difference. (c) Interestingly in the complete-chemotherapy group, there was no difference in high and low SDF1a amplification toward to metastasis

Table 4. The Duration to Distant Metastasis and Survival (in Months) Difference between High and Low Amplification of SDF1a, and Expression of Cytoplasm and NuAclear

\begin{tabular}{|c|c|c|c|c|c|}
\hline Variables & & $n$ & Mean & SD & $\mathrm{p}$ \\
\hline \multirow[t]{6}{*}{ SDF1a } & Month to distant metastasis & & & & \\
\hline & Low & $64(49 \%)$ & 35.98 & 28.39 & $<0.001$ \\
\hline & High & $67(51 \%)$ & 19.96 & 16.28 & \\
\hline & Survival in month & & & & \\
\hline & Low & $64(49 \%)$ & 41.39 & 29.25 & $<0.001$ \\
\hline & High & $67(51 \%)$ & 25.49 & 18.46 & \\
\hline \multirow[t]{6}{*}{ CXCR4 Cytoplasma } & Month to distant metastasis & & & & \\
\hline & Low & $117(87 \%)$ & 28.50 & 25.05 & 0.537 \\
\hline & High & $17(13 \%)$ & 24.53 & 21.20 & \\
\hline & Survival in month & & & & \\
\hline & Low & $117(87 \%)$ & 33.61 & 26.44 & 0.719 \\
\hline & High & $17(13 \%)$ & 31.18 & 21.84 & \\
\hline \multirow[t]{6}{*}{ CXCR4 Nuclear } & Month to distant metastasis & & & & \\
\hline & Low & $37(30 \%)$ & 28.35 & 23.23 & 0.831 \\
\hline & High & $87(70 \%)$ & 27.32 & 25.12 & \\
\hline & Survival in month & & & & \\
\hline & Low & $37(30 \%)$ & 32.08 & 21.80 & 0.772 \\
\hline & High & $87(70 \%)$ & 33.56 & 27.51 & \\
\hline
\end{tabular}



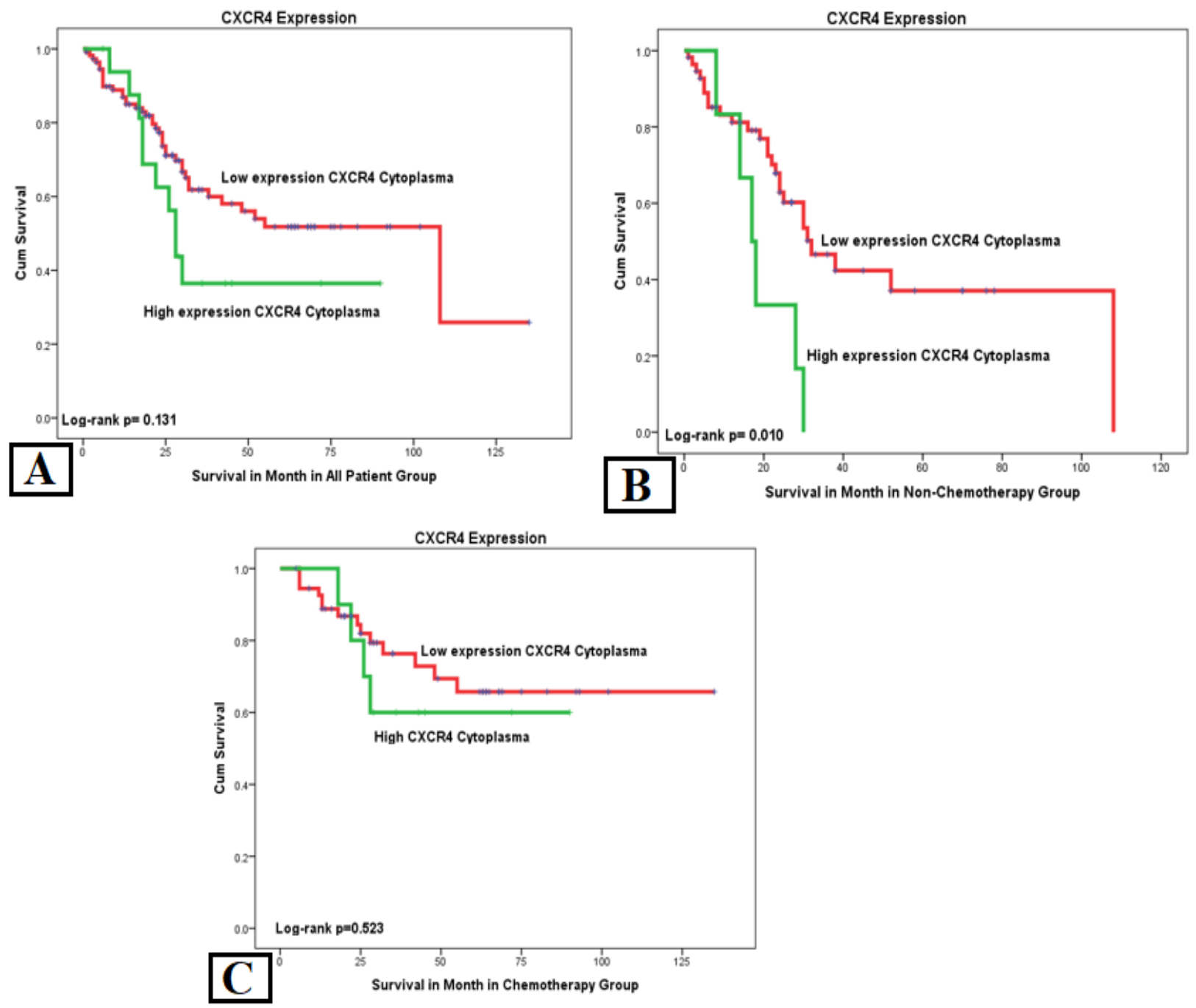

Figure 4. Kaplan-Meier Chart of Cytoplasmic CXCR4 to Overall Survival in All 3 Patient Groups. (a) High cytoplasmic CXCR4 expression showed shorter metastatic time although it was not significant in the all-patient group. (b) High cytoplasmic CXCR4 expression in the no chemotherapy group showed a significant difference in the poor OS with $\mathrm{P}=0.010$. (c) But interestingly complete-chemotherapy group showed no significant difference between high and low cytoplasmic CXCR4 expressions

receptor, showed a protective effect on OS with $\mathrm{p}=0.019$ $\mathrm{HR}=0.354$. The complete-chemotherapy group showed that only stage was correlated with OS with $\mathrm{p}=0.020$ $\mathrm{HR}=3.823$. There was no correlation of SDF1a towards OS in the complete-chemotherapy group (Table 3 ).

Stage has the strongest correlation in all-patient, no-chemotherapy, and chemotherapy groups, both on the distant metastasis and the OS. In Kaplan-Meier curve analysis, we found that higher expression of cytoplasmic CXCR4 and SDF 1a expression was related with poor OS in the no-chemotherapy group (Figure 4 and 5). Patients with high SDF1a amplification have a shorter mean OS of 25.49 months than low SDF1a amplification with a mean OS of 41.39 months and shorter time to distant metastasis of 19.96 months compared to low SDF1a amplification with a mean of 35.98 months (Table 4).

\section{Discussion}

In our previous study, the expression of $S D F 1 a$ with $I H C$ was higher in groups with distant metastasis.
Expressions include cancer cells as autocrine and other cells in the tumor microenvironment as paracrine (Yarso et al., 2016). This process can affect through two mechanisms: first, by direct stimulation of SDF1a to cancer cells that will induce growth and motility to avoid apoptosis and, second, by influencing the microenvironment, among them, by attracting EPC that induces angiogenesis so that cancer cells can intravasate more easily (Asri et al., 2016).

In this study, SDF1a correlated with worse in distant metastases and OS in all-patient and no-chemotherapy groups, but not in the chemotherapy group. Cytoplasmic and nuclear CXCR4 did not have any correlation between metastasis and OS in all-patient, no-chemotherapy, and chemotherapy group. The hormone receptor has a protective effect on OS in the no-chemotherapy group even though advanced stage is continually showing a significant correlation with metastasis and OS in allpatient, no-chemotherapy, and chemotherapy group.

In developing countries like Indonesia, trust in alternative medicine, fear of hair loss, and wrong 

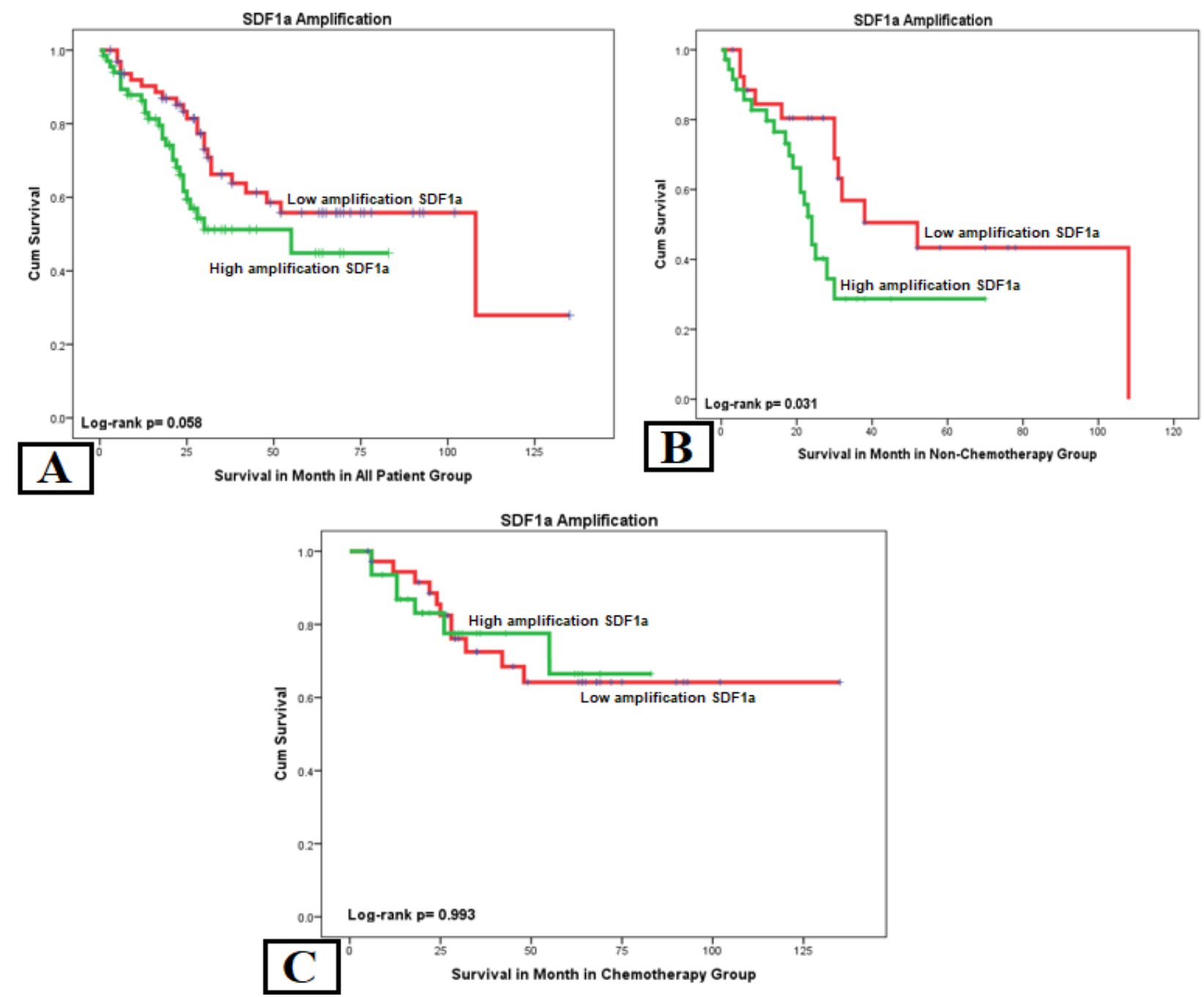

Figure 5. Kaplan-Meier Chart of SDF1a to Overall Survival in All 3 Patient Groups. (a) The SDF1 amplification showed a no significant OS in the all-patient group. (b) High expression of SDF 1 in no-chemotherapy group had significantly different OS. (c) Complete-chemotherapy group showed no significant difference between high and low SDF1a

myths about chemotherapy cause not all patients to undergo or drop out of chemotherapy. Refusing standard chemotherapy alone will reduce survival by more than $30 \%$. Verkooijen (2005) found a decrease of 36\% vs. $75 \%$ in 10 years. Meanwhile, Joseph (2012) found poor outcomes compared to patients receiving standard chemotherapy by $43.2 \%$ vs. $81.9 \%$. From a previous study, administering GSCMF or cyclophosphamide reported decreased SDF-1 concentrations in vivo in rat bone marrow and decreased the accumulation of serine protease, which can directly cleave and regulate SDF-1 proteolytic degradation of SDF-1, along with CXCR4 (Levesque et al., 2003). Unfortunately, this research did not lead to clinical study. This study may answer why patients with high SDF1a in the complete-chemotherapy group are not associated with OS and metastases. In other breast cancer studies with dense chemotherapy with GSCMF, it is believed that dose-dense treatment may partly reflect the inhibition of micrometastasis homing and/or paracrine survival associated with CXCR4 (Epstein, 2004).

Previous research stated that the expression of the SDF1 score from IHC might be higher in subjects with metastasis. Other research also shows IHC from SDF1 is associated with poor survival and local recurrence, similar to the study of transcription of frozen section tissue using IHC and RtPCR (Kang et al., 2005). In contrast to Italy, Mirisola et al., (2009) stated SDF1a with microarrays and IHC was associated with good DFS and OS. Besides, SDF1 in cancerous tissue can also induce aggressiveness by calling on EPC or modification of the immune system by recruiting lymphocytes (Petit et al., 2007).

The secretion of CXCL12 by breast cancer cells can enhance invasion, recruit macrophages, and increase microvessel density, which may also be mediated by tumor-associated macrophages and contributes to altered tumor architecture. These results demonstrate how a tumor can increase invasion and motility and contribute to enhanced tumor malignancy (Boimel et al., 2012).

Preliminary study in Indonesia about $C X C R 4$ with $I H C$, the expression is the same for breast cancer with or without distant metastasis, but the high expression of CXCR4 tends to have metastases to the lungs other organs (Hariyanto, 2012). In China, high nuclear CXCR4 was associated with negative lymph node metastases, while high cytoplasmic $C X C R 4$ expression was associated with patients with lymph node metastases (Su et al., 2006). 
These results contradict the previous study in Korea, which found that high $C X C R 4$ expression in the cytoplasm was associated with better RFS and OS in triple-negative breast cancer with chemotherapy (Shim et al., 2018).

Theoretically, the high CXCR4 outcomes have more aggressive metastases both in-vitro and in-vivo (Hernandez et al., 2011). In CXCR4 receptors, there are still proteins that affect the sensitivity of the stimulation from SDF1, including GRK and Arestin 2 proteins, influencing the active cascade under CXCR4. Another study has shown a decrease in the sensitivity of CXCR4 to induction from SDF1 by performing endocytosis and degradation (Bushillo et al., 2010).

In the study using stromal mesenchymal cells from the fetus, CXCR 4 was only found in the membrane by $4 \%$. The rest in the endosome or lysosome might be at the stage of CXCR4 recycling. There was also CXCR4 in the cell nucleus. The treatment that increases the expression of CXCR4 in the membrane turns out to increase metastasis 2.6 times (Pelekanos et al., 2014).

In conclusion, SDF1a mRNA amplification has a significant correlation with the occurrence of metastasis and OS in all-patient and no-chemotherapy group. Undergoing chemotherapy negates the effect of SDF1a for distant metastasis and OS.

\section{Author Contribution Statement}

Kristanto Yuli Yarso: funding, data collection, statistic, primary investigation, supervision, writing; Monica Bellynda: data collection, manuscript, software, writing; Akhmad Azmiardi: format analysis, software; Brian Wasita: data collection (histopathological), data curation; Didik Setyo Heriyanto: data curation, rtPCR; Indwiani Astuti, Mohammad Hakimi: methodology; Teguh Aryandono: conceptualization.

\section{Acknowledgements}

This article is part of an approved dissertation.

\section{Ethical Approval}

This study was reviewed and approved by the ethics committee of the Medical Faculty of Gadjah Mada University Reference number: KE/F/35/EC.

\section{Declaration of Interest}

The authors declare no conflict of interest

\section{References}

Arima N, Nishimura R, Osako T, et al (2019). Ki-67 index value and progesterone receptor status can predict prognosis and suitable treatment in node-negative breast cancer patients with estrogen receptor-positive and HER2-negative tumors. Oncol Lett, 17, 616-22.

Asri A, Sabour J, Atashi A, et al (2016). Homing in hematopoietic stem cells: focus on regulatory role of CXCR7 on SDF1a/ CXCR4 axis. Excli J, 15, 134-43.

Boimel PJ, Smirnova T, Zhou ZN, et al (2012). Contribution of CXCL12 secretion to invasion of breast cancer cells. Breast Cancer Res, 14, R23.
Busillo JM, Armando S, Sengupta R, et al (2010). Site-specific phosphorylation of CXCR4 is dynamically regulated by multiple kinases and results in differential modulation of CXCR4 signaling. J Biol Chem, 285, 7805-17.

Epstein RJ (2004). The CXCL12-CXCR4 chemotactic pathway as a target of adjuvant breast cancer therapies. Nat Rev Cancer, 4, 901-9.

Fitzgibbons PL, Page DL, Weaver D, et al (2000). Prognostic factors in breast cancer. College of American Pathologists Consensus Statement 1999. Arch Pathol Lab Med, 124, 966-78.

Hariyanto G (2012). Metastasis paru berhubungan dengan over ekspresi chemokine receptor 4 tumor primer pada karsinoma payudara. Thesis, Universitas Udayana, Bali.

Hernandez L, Magalhaes MAO, Coniglio SJ, et al (2011). Opposing roles of CXCR4 and CXCR7 in breast cancer metastasis. Breast Cancer Res, 13, R128.

Joseph K, Vrouwe S, Kamruzzaman A, et al (2012). Outcome analysis of breast cancer patients who declined evidencebased treatment. World J Surg Oncol, 10, 118.

Kang H, Escudero-Esparza A, Douglas-Jones A, et al (2009). Transcript analyses of stromal cell derived factors (SDFs): SDF-2, SDF-4 and SDF-5 reveal a different pattern of expression and prognostic association in human breast cancer. Int J Oncol, 35, 205-11.

Kang H, Watkins G, Parr C, et al (2005). Stromal cell derived factor-1: its influence on invasiveness and migration of breast cancer cells in vitro, and its association with prognosis and survival in human breast cancer. Breast Cancer Res, 7, R402-10.

Kim JO, Suh KS, Lee DH, et al (2009). Expression of CXCR4 and SDF- $1 \alpha$ in primary breast cancers and metastatic lymph nodes. J Breast Cancer, 12.

Lévesque JP, Hendy J, Takamatsu Y, et al (2003). Disruption of the CXCR4/CXCL12 chemotactic interaction during hematopoietic stem cell mobilization induced by GCSF or cyclophosphamide. J Clin Invest, 111, 187-96.

Liu F, Lang R, Wei J, et al (2009). Increased expression of SDF-1/ CXCR4 is associated with lymph node metastasis of invasive micropapillary carcinoma of the breast. Histopathology, 54, 741-50.

Mirisola V, Zuccarino A, Bachmeier BE, et al (2009). CXCL12/ SDF1 expression by breast cancers is an independent prognostic marker of disease-free and overall survival. Eur J Cancer, 45, 2579-87.

Pelekanos RA, Ting MJ, Sardesai VS, et al (2014). Intracellular trafficking and endocytosis of CXCR4 in fetal mesenchymal stem/stromal cells. BMC Cell Biol, 15, 15.

Petit I, Jin D, Rafii S (2007). The SDF-1-CXCR4 signaling pathway: a molecular hub modulating neo-angiogenesis. Trends Immunol, 28, 299-307.

Shim B, Jin MS, Moon JH, et al (2018). High cytoplasmic CXCR4 expression predicts prolonged survival in triplenegative breast cancer patients treated with adjuvant chemotherapy. J Pathol Transl Med, 52, 369-77.

Su JL, Yang PC, Shih JY, et al (2006). The VEGF-C/Flt-4 axis promotes invasion and metastasis of cancer cells. Cancer Cell, 9, 209-23.

Su YC, Wu MT, Huang CJ, et al (2006). Expression of CXCR4 is associated with axillary lymph node status in patients with early breast cancer. Breast $J, 15,533-9$.

Verkooijen HM, Fioretta GM, Rapiti E, et al (2005). Patients' refusal of surgery strongly impairs breast cancer survival. Ann Surg, 242, 276-80.

Verkooijen HM, Fioretta GM, Rapiti E, et al (2005). Patients' refusal of surgery strongly impairs breast cancer survival. Ann Surg, 242, 276-80. 
Woo SU, Bae JW, Kim CH, et al (2008). A significant correlation between nuclear CXCR4 expression and axillary lymph node metastasis in hormonal receptor negative breast cancer. Ann Surg Oncol, 15, 281-5.

World Health Organization (WHO) (2019). Indonesia Fact Sheets - World Health Organization. https://gco.iarc.fr/ today/data/factsheets/populations/360-indonesia-fact-sheets. pdf.

Yarso KY, Sudarsa IW, Wibawa-Manuaba IBT (2012). Clinical initial response of neoadjuvant chemotheraphy in triple negative, HER-2, and luminal types of breast cancer in denpasar (A Preliminary Study). Bali Med J, 1.

Yarso KY, Suyatmi S, Syah FK, et al (2016). Association of SDF-1 with Metastasis in Breast Cancer Patient at Sanglah Hospital, Bali. Bali Med J, 5, 134.

Yasuoka H, Tsujimoto M, Yoshidome K, et al (2008). Cytoplasmic CXCR4 expression in breast cancer: induction by nitric oxide and correlation with lymph node metastasis and poor prognosis. BMC Cancer, $\mathbf{8}, 340$.

This work is licensed under a Creative Commons AttributionNon Commercial 4.0 International License. 